\title{
Estimación de parámetros operativos eficientes enfocados a la fabricación de bobinas eléctricas empleando un diseño experimental $2^{k}$ Estimation of efficient operating parameters focused to the manufacture of electric coils using an experimental design $2^{\mathrm{k}}$
}

Edgar Augusto Ruelas Santoyo ${ }^{1}$ y José Jovani Cardiel Ortega ${ }^{1}$

Palabras clave: bobinas eléctricas; industria eléctrica; diseño de experimentos Keywords: electric coils; electrical industry; design of experiments

Recibido en: 17-10-2018 / Aceptado en: 29-05-2019

\section{Resumen}

Introducción: Ante la importancia de la industria eléctrica del país por incidir en la mayoría de los procesos productivos de la economía, los retos en los cambios en el mercado energético y las nuevas regulaciones para los productores, surge la necesidad, desde el aspecto de la manufactura de ofrecer herramientas para mejorar los procesos de fabricación de productos eléctricos, la calidad del servicio y aumentar la confiabilidad del sistema. El transformador es uno de los elementos más importantes en el sistema de transmisión de energía, fabricarlo con bajos estándares de calidad representa problemas importantes tanto para los consumidores finales como para el sistema en general. El propósito de este trabajo es establecer condiciones de operación eficientes para la manufactura de bobinas eléctricas, minimizando la variabilidad de las dimensiones y permitir un correcto ensamble de los componentes que integran el transformador monofásico.

Método: Se utilizó el análisis de capacidad para evaluar la habilidad del proceso al cumplir con las especificaciones antes y después de las mejoras, además, se desarrolló un Diseño de Experimentos Factorial $2^{k}$ que permitió establecer condiciones de operación adecuadas en el proceso de manufactura además de determinar la influencia de la tensión del alambre y el proveedor del papel dieléctrico sobre las variables dimensionales de la bobina.

Resultados: Con el estudio se encontró que los factores considerados tienen influencia significativa sobre las dimensiones de las bobinas, los niveles altos de cada factor permitieron minimizar las dimensiones, con ello fue posible cumplir con los límites de especificación, se logró mejorar el centrado del proceso en las variables del largo y ancho de la bobina al incrementar sustancialmente los índices de capacidad reales del proceso.

\footnotetext{
${ }^{1}$ Instituto Tecnológico Superior de Irapuato. Irapuato, Guanajuato. E-mail: edruelas@itesi.edu.mx (C) Universidad De La Salle Bajío (México)
} 
Discusión o Conclusión: Los métodos estadísticos utilizados en este trabajo fueron una herramienta eficaz para estudiar el proceso, minimizar las dimensiones, mejorar la eficiencia del producto y los costos por re-trabajos.

\begin{abstract}
Introduction: Given the importance of the country's electricity industry for influencing most of the productive processes of the economy, the challenges in the changes in the energy market and the new regulations for producers, it is necessary, from the aspect of manufacturing to offer tools to improve the processes of electrical products, the quality of the service and increase the reliability of the system. The transformer is one of the most important elements in the electric power transmission system, manufacturing it with low quality standards represents important problems for both the final consumers and the system. The purpose of this work is to establish efficient operating conditions for the manufacture of electric coils, minimizing the variability of the dimensions and allowing a correct assembly of the components that make up the single-phase transformer.
\end{abstract}

Method: Capacity analysis was used to evaluate the ability of the process to meet the specifications before and after the improvements, in addition, a $2^{\mathrm{k}}$ Factorial Experiments Design was developed that allowed to establish adequate operating conditions in the manufacturing process in addition to determine the influence of the tension of the wire and the dielectric paper supplier on the dimensional variables of the coil.

Results: With the study it was found that the factors considered have significant influence on the dimensions of the coils, the high levels of each factor allowed to minimize the dimensions, thus it was possible to meet the specification limits, it was possible to improve the centering of the process of the variables of length and width of the coil by substantially increasing the capability indices of the process.

Discussion or Conclusion: The statistical methods used in this work were an effective tool to study the process. 


\section{Introducción}

La electricidad es un bien preferente que no admite sustitutos, presenta una demanda casi perfectamente inelástica al precio, no es almacenable a gran escala (lo que prohíbe crear provisiones a mediano y largo plazo), es prácticamente imposible (técnica y económicamente) satisfacer la demanda eléctrica con una sola tecnología y además, incide en la mayoría de los procesos productivos de la economía (Payan, 2016). Según las últimas proyecciones del Consejo Nacional de Población (CONAPO, 2010), México tendrá en 2030 una población de 137.5 millones de personas, que en su momento, tendrá un consumo bruto eléctrico de 445,301.8 GWh (SENER, 2017). Es decir, en 2030 se consumirá 45.4\% más energía eléctrica que en 2017 (Limón, 2017). La red de distribución es la infraestructura que transporta la energía eléctrica generada por grandes plantas alejadas del usuario final, consiste en centros de transformación de alto a medio voltaje y de medio a bajo voltaje. Los clientes residenciales y comerciales están principalmente conectados al bajo voltaje, mientras que las grandes instalaciones industriales están conectadas al medio voltaje, (ver Fig. 1). En los últimos años, los sistemas de distribución se han visto afectados por muchos cambios significativos. La desregulación del mercado energético, la creciente penetración de la generación distribuida, principalmente fuentes de energía renovables, y los nuevos recursos de energía distribuidos tales como los vehículos eléctricos, han transformado la red pasiva en una red activa altamente compleja. Estos aspectos, junto con los requisitos siempre más estrictos para mejorar la calidad de la energía y la confiabilidad del sistema, requieren una gestión y un control más efectivos de la red (Della et al., 2014).

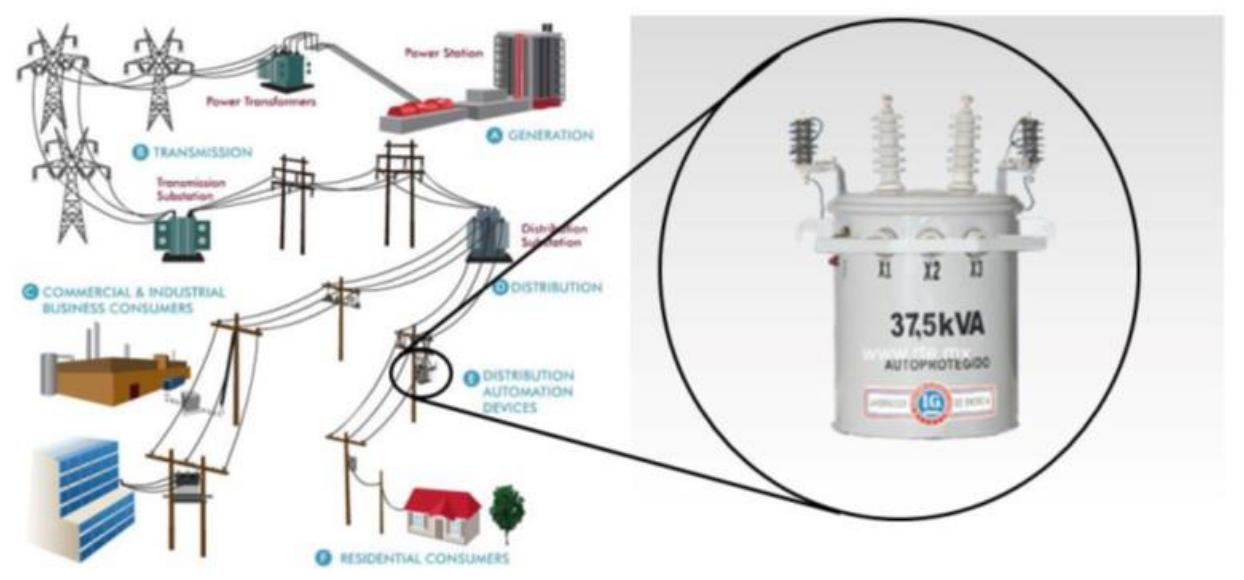

Fig. 1. Sistema de distribución eléctrico. 
En las últimas décadas el sector eléctrico a nivel mundial ha sufrido grandes cambios en materia regulatoria, siempre con miras a mejorar la calidad del servicio y la potencia suministrada al usuario final. Para esto se han elaborado leyes que rigen el mercado de energía y el servicio, definiendo los límites permisibles para las variables y características del servicio. Con el objetivo de cumplir con las restricciones regulatorias y hacer uso eficiente de los recursos disponibles, las empresas de distribución han dedicado esfuerzos y recursos en planear la forma como el sistema debe estar preparado para atender contingencias operativas y disponer de capacidad suficiente para atender el crecimiento de la demanda, todo esto bajo condiciones de costo-eficiencia y características técnicas básicas (García et al., 2015).

Lo anterior, no solo resalta la importancia que tiene la industria eléctrica en el ámbito económico, sino también en aspectos como la confiabilidad del servicio y la calidad de los productos utilizados en la red de distribución de energía, con la finalidad de ofrecer una plena satisfacción al consumidor final. La principal propuesta de este artículo la estimación de parámetros de operación eficientes para las variables con mayor influencia en la fabricación de bobinas eléctricas utilizadas en los transformadores, se utilizó la herramienta de Diseño de experimentos (DOE, por sus siglas en inglés) y el análisis de capacidad.

El diseño de experimentos y los métodos estadísticos han sido aplicados con el fin de implementar condiciones de operación adecuadas en la manufactura de transformadores eléctricos de distintos tipos, parte de la literatura revisada se presenta a continuación.

Ranade et al. (2016), realizaron un estudio para optimizar un taque de transformador 6.6 MVA 66/11.5 KV con el fin de reducir el peso del tanque, utilizaron el método de elementos finitos (MEF), además, la metodología de cribado, diseño central compuesto y superficie de respuesta. A través del software ANSYS simularon el diseño, el análisis y la optimización. Sus resultados demuestran que aproximadamente el $15 \%$ del peso se reduce manteniendo la deformación y las tensiones por debajo del límite.

Dolan y Stefanescu (2016), aplicaron un método exhaustivo de optimización basado en DOE y el MEF en un superconductor de almacenamiento de energía magnética, eligieron dos parámetros geométricos, la relación de diámetro interno de la bobina y la relación de espesor de la bobina. El algoritmo de optimización se basó en experimentos numéricos 2-D para calcular modelos polinominales de segundo orden de la función objetivo utilizando diseño factorial 
completo con tres niveles por factor. El resultado es el máximo global y se compara con soluciones anteriores.

Zitouni et al. (2016), desarrollaron un diseño experimental factorial en laboratorio para modelar el voltaje de ruptura, en las pruebas utilizaron un transformador de alto voltaje, un divisor de voltaje capacitivo y una celda de prueba transparente, estudiaron la influencia de los parámetros geométricos (la distancia entre los electrodos, la posición relativa de la barrera y de cada diámetro), principalmente, el diseño permitió reducir el número de pruebas de laboratorio minimizando los costos. Por su parte, Dessouky et al. (2017), en su trabajo utilizan metodología de superficie de respuesta basada en el diseño de Box-Benken para construir una fórmula de predicción y estudiar el impacto del espacio vacío con la barrera entre dos placas, la barrera colocada entre las dos placas relativas al espacio de separación y eventualmente, el diámetro de la barrera sobre la resistencia a la ruptura de los aceites aislantes de los transformadores. Los resultados explican la capacidad del método propuesto para dar una fórmula de predicción con un número mínimo de experimentos requeridos y errores mínimos, además, de la optimización de los parámetros estudiados. Los resultados estadísticos refieren que el diámetro de la barrera no tiene un efecto significativo sobre la tensión de ruptura.

Ruelas-Santoyo et al. (2018), implementaron la carta de control $\mathrm{T}^{2}$ de Hotelling como mecanismo de control en procesos multivariantes, en una línea de producción de trasformadores eléctricos del tipo monofásico con una potencia de $25 \mathrm{KVA}$, para establecer un adecuado control estadístico del proceso del ensamble de la bobina y la parte activa se monitorearon tres características de calidad, largo en la bobina, el ancho del núcleo y largo del núcleo.

Heidari-Kapourchali et al. (2016), presentan un diseño factorial de $2^{4}$ para analizar el efecto de la carga conjunta, las características de enfriamiento, la temperatura ambiente y la constante de tiempo del aceite en la pérdida de vida del transformador. Los resultados muestran cómo el diseño del experimento ayuda a encontrar la mejor combinación de factores y sus niveles en la pérdida de vida de los transformadores de distribución para mejorar la eficiencia de la red.

Petkovska et al. (2014), proponen un enfoque para la optimización del diseño multiobjetivo de un transformador de distribución con el fin de aumentar la eficiencia al reducir la pérdida total y minimizar la masa de hierro y cobre y, por lo tanto, lograr un producto nuevo, más eficiente y de menor costo. Emplearon el método Taguchi y DOE, sus resultados, tanto 
analíticos como numéricos fueron validados a través de mediciones de un nuevo prototipo, demostraron que el nuevo enfoque fue efectivo y preciso.

Phaengkieo et al. (2013), en su investigación desarrollan un diseño de experimentos central compuesto y utilizan el MEF con el objetivo de optimizar parámetros como el grosor del núcleo, el número de vueltas del devanado y el área de conducción para obtener mejores rendimientos de los transformador eléctricos. Con lo anterior logran mejorar la eficiencia, pérdida, temperatura y particularmente el costo del transformador.

Como es posible observar, el uso de herramientas estadísticas y en muchos casos el complementar con técnicas matemáticas y de simulación permite mejorar la calidad de los transformadores con el fin de reducir las fallas, mejorar los procesos de diseño y fabricación, así como, disminuir los costos y aumentar la eficiencia energética.

Según la secretaria de energía (SENER, 2017), la industria eléctrica se compone de 4 actividades; generación, trasmisión, distribución y comercialización. Los transformadores eléctricos entran en los productos de transmisión. Un transformador es uno de los elementos más importantes de un sistema de transmisión de energía eléctrica. Una falla en el transformador puede presentar problemas importantes tanto para los consumidores como para los ingenieros de mantenimiento (Al-Janabi et al., 2015).

El transformador eléctrico es una máquina estática compuesta en esencia por al menos dos bobinas o devanadas denominadas bobina primaria y bobina secundaria (ver Fig. 2). El transformador tiene como función cambiar en nivel de tensión de una bobina con respecto a la otra, bien sea disminuyéndola o aumentándola, cuya variación de tensión está ligada a la relación de transformación o número de espiras entre bobinas. El principio de funcionamiento de los transformadores depende de la variación de flujo dentro de un circuito magnético para producir el efecto de inducción magnética y con ello cambiar los niveles de tensión eléctrica (Anteliz, 2012).

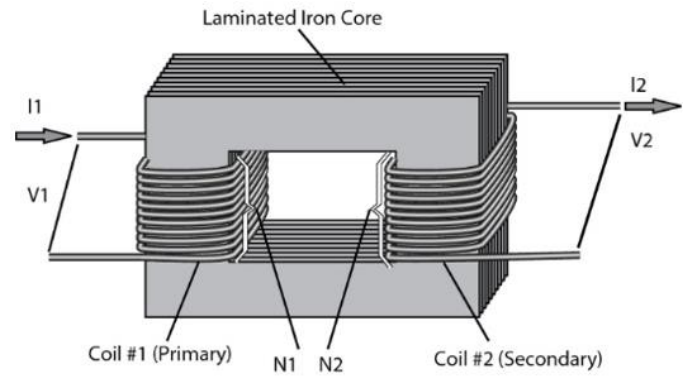

Fig. 2. Representación de un transformador eléctrico.

Fuente: Hydraulics \& Pneumatics, 2018.

№ 23, Vol. 11 (2), 2019. ISSN 2007 - 0705, pp.: 26 - 48 
En este trabajo se propone establecer parámetros de operación eficientes para la fabricación de bobinas monofásicas y minimizar la variabilidad de las dimensiones con el fin de tener un correcto ensamble de los componentes que integran al transformador. El estudio se enfocó en transformadores monofásicos tipo poste con una potencia de 15kVA, además, se llevó a cabo, un estudio de capacidad previo al diseño experimental y posterior al implementar los parámetros eficientes de fabricación seleccionados.

Una de las etapas importantes durante el proceso de manufactura de los transformadores eléctricos es el conjunto de parte viva, que consiste en el ensamble de la bobina con el núcleo; después, la parte viva es ensamblada en el tanque del transformador, con ello se tienen los componentes principales para continuar con el proceso y contar con el producto final, (ver Fig. $3)$.
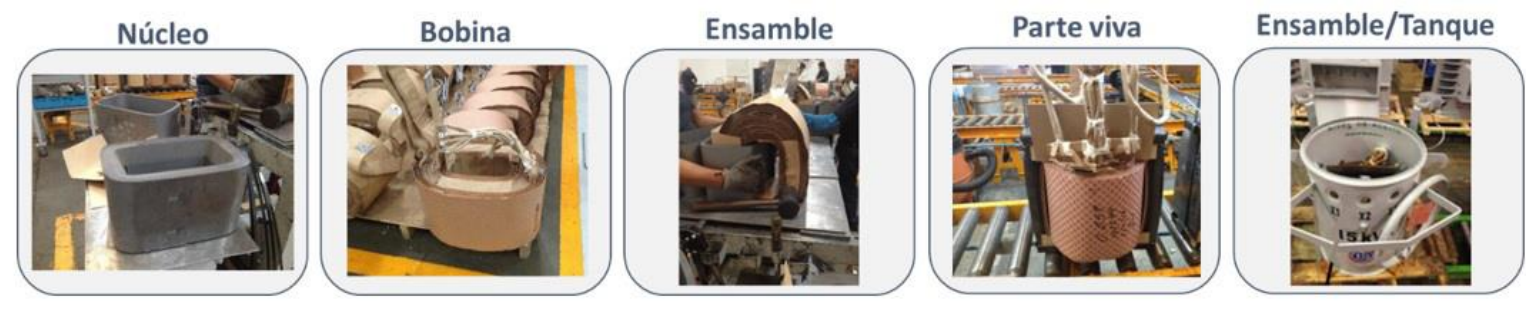

Fig. 3. Ensamble transformador monofásico.

Con la entrada en vigor de la Norma Oficial Mexicana NOM-002-SEDE/ENER-2014 (Requisitos de seguridad y eficiencia energética para transformadores de distribución), permanecer en el mercado del sector eléctrico es cada vez más complicado y exigente, y para dar respuesta a esta demanda se han generado estrategias para incrementar la competitividad en el mercado, como lo es la necesidad de manufacturar productos con los niveles más altos de calidad y procesos más competitivos.

\section{Metodología}

Para lograr el propósito del estudio y partiendo de la observación sistemática del proceso, se realizó la metodología descrita en la Tabla 1, en donde se presentan diez etapas, los objetivos y los medios utilizados para lograrlos. 
Tabla 1. Metodología de investigación.

\begin{tabular}{|lll|}
\hline \multicolumn{1}{|c|}{ Etapa } & \multicolumn{1}{c|}{ Objetivo } & \multicolumn{1}{c|}{ Medios utilizados } \\
\hline $\begin{array}{l}\text { Análisis del } \\
\text { proceso }\end{array}$ & $\begin{array}{l}\text { Detectar la problemática principal en la fabricación } \\
\text { de transformadores para tomar acciones de mejora }\end{array}$ & Observación sistemática \\
\hline $\begin{array}{l}\text { Revisión de la } \\
\text { literatura }\end{array}$ & $\begin{array}{l}\text { Consultar trabajos previos relacionados con el } \\
\text { estudio de boninas para complementar el } \\
\text { conocimiento sobre el proceso en estudio }\end{array}$ & $\begin{array}{l}\text { Base de datos de artículos } \\
\text { científicos }\end{array}$ \\
\hline Marco teórico & $\begin{array}{l}\text { Profundizar en la herramienta de diseño de } \\
\text { experimentos para su correcta implementación }\end{array}$ & Bibliografía \\
\hline $\begin{array}{l}\text { Estudio de } \\
\text { capacidad inicial }\end{array}$ & $\begin{array}{l}\text { Determinar la variación del proceso con el fin de } \\
\text { revisar sus causas }\end{array}$ & $\begin{array}{l}\text { Cartas de control, histogramas e } \\
\text { índices de capacidad }\end{array}$ \\
\hline $\begin{array}{l}\text { Planeación del } \\
\text { diseño }\end{array}$ & $\begin{array}{l}\text { Diseñar el plan experimental para determinar el } \\
\text { diseño adecuado, corridas, tratamientos y } \\
\text { procedimiento a seguir }\end{array}$ & $\begin{array}{l}\text { Análisis por medio de reuniones de } \\
\text { las áreas de manufactura, ingeniería }\end{array}$ \\
\hline $\begin{array}{l}\text { Ejecución del } \\
\text { diseño }\end{array}$ & $\begin{array}{l}\text { Llevar acabo la experimentación para conocer los } \\
\text { factores significativos y obtener mayor } \\
\text { conocimiento sobre el proceso }\end{array}$ & y calidad \\
\hline $\begin{array}{l}\text { Análisis } \\
\text { estadístico }\end{array}$ & $\begin{array}{l}\text { Analizar los resultados para determinar los mejores } \\
\text { tratamientos que mejoren la variable de respuesta }\end{array}$ & $\begin{array}{l}\text { Análisis de varianza, graficas de } \\
\text { efectos, interacción y supuestos }\end{array}$ \\
\hline Validación & $\begin{array}{l}\text { Implementar lo resultados en planta para confirmar } \\
\text { los resultados de la investigación }\end{array}$ & $\begin{array}{l}\text { Corridas de confirmación } \\
\text { (fabricación) }\end{array}$ \\
\hline $\begin{array}{l}\text { Estudio de } \\
\text { capacidad final }\end{array}$ & $\begin{array}{l}\text { Determinar la variación del proceso para evaluar la } \\
\text { habilidad del proceso de cumplir con las } \\
\text { especificaciones }\end{array}$ & $\begin{array}{l}\text { Cartas de control, histogramas e } \\
\text { índices de capacidad }\end{array}$ \\
\hline $\begin{array}{l}\text { Resultados y } \\
\text { conclusiones }\end{array}$ & $\begin{array}{l}\text { Evaluar los logros para ofrecer conclusiones } \\
\text { basadas en los hallazgos }\end{array}$ & Evaluación de los datos \\
\hline
\end{tabular}

\section{Desarrollo}

Los transformadores generan una de las mayores pérdidas en el sistema y en la red de distribución de energía, por ello en la actualidad surge la necesidad de que la industria eléctrica genere productos con mayor eficiencia y calidad, pero al mismo tiempo logren reducir sus costos de fabricación para ser competitivos y sin afectar al consumidor final.

El incremento en la eficiencia causa de manera inherente mayor utilización de material en la fabricación, por lo tanto, el precio del transformador puede llegar a elevarse en un porcentaje considerable en función de la clase de aislamiento y potencia.

En la línea de producción de la empresa en estudio se encontró que existían problemas en el ensamble del conjunto de parte viva y a su vez de la parte viva al tanque, ocasionando retrabajos y desperdicio de materiales representando un incremento en el costo total del transformador.

El grupo de trabajo interdepartamental integrado por las áreas de producción, control de calidad, ingeniería de diseño y de manufactura, además, del apoyo del personal operativo (bobinadores y ayudantes de bobinadores) determinó que la bobina era el principal problema para 
el ensamble del conjunto de la parte viva, por ello es fundamental lograr establecer parámetros eficientes de fabricación en el proceso.

\section{1) Análisis de capacidad inicial}

Evaluar la habilidad de un proceso consiste en conocer la amplitud de la variación natural de éste para una característica de calidad dada, lo cual permitirá saber en qué medida tal característica es satisfactoria (Gutiérrez y de la Vara Salazar, 2013).

Tanto el componente del núcleo, como las bobinas definen el tamaño final de los transformadores, el criterio de diseño para la fabricación de las bobinas establece que las especificaciones de los límites de fabricación en sus dimensiones finales deben ser de $\pm 5 \%$ del valor nominal en el ancho y largo. La estructura física de la bobina se muestra en la Fig. 4.

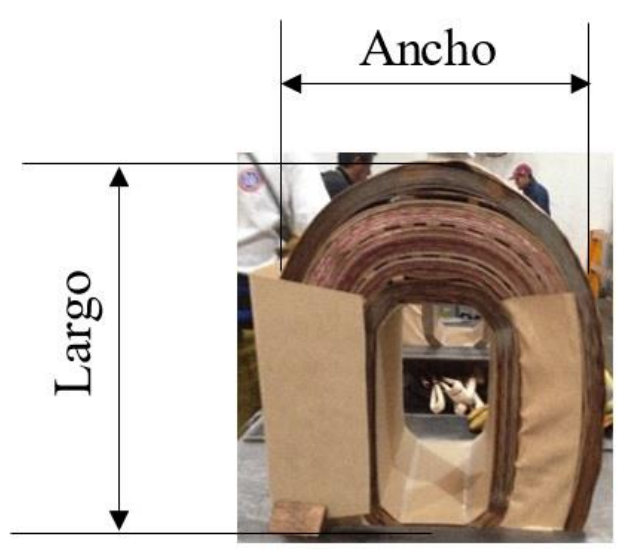

Fig. 4. Variables dimensionales.

Para corroborar el impacto de la bobina en el ensamble de parte viva se realizó un estudio de capacidad del proceso. Los límites dimensionales definidos para la fabricación se presentan en la Tabla 2.

Tabla 2. Límites dimensionales.

\begin{tabular}{|c|cc|}
\hline Límites & Ancho & Largo \\
\hline Superior & $262.5 \mathrm{~mm}$ & $404.3 \mathrm{~mm}$ \\
Control & $250.0 \mathrm{~mm}$ & $385.0 \mathrm{~mm}$ \\
Inferior & $237.5 \mathrm{~mm}$ & $365.8 \mathrm{~mm}$ \\
\hline
\end{tabular}

№ 23, Vol. 11 (2), 2019. ISSN 2007 - 0705, pp.: 26 - 48 
Se realizaron mediciones de las variables de ancho y largo a partir de un total de 140 bobinas correspondientes a un mes de producción del modelo de transformador seleccionado, el volumen de producción por mes es variable, con un promedio de 150 transformadores fabricados en total. De acuerdo con (Montgomery y Runger, 2014), el tamaño de la muestra puede ser determinado a través de la (Ecuación 1):

$$
\mathrm{n}=\frac{\mathrm{n}_{0}}{1+\frac{\left(\mathrm{n}_{0}+1\right)}{\mathrm{N}}}
$$

Ecuación 1. Tamaño de la muestra estadística.

Donde:

N: Tamaño de la población

$\mathrm{n}_{\mathrm{o}}$ : Tamaño de la muestra para una población finita

$\mathrm{n}_{\mathrm{o}}=\frac{\mathrm{z}_{\alpha / 2}^{2} * \mathrm{p} * \mathrm{q}}{\mathrm{e}^{2}}$

p: Proporción

q: Proporción complementaria (1- p)

e: Margen de error

z: Nivel de confianza estandarizado

El tamaño de la muestra queda definido en la (Ecuación 2), tomando el tamaño de la población como el volumen de producción promedio mensual de 150 transformadores, el nivel de confianza empleado es del 95\% ( $\mathrm{z}=1.96)$, una proporción del 50\%, y un margen de error del 3\%. El tamaño de muestra propuesto es más pequeño que el empleado, por lo tanto el análisis estadístico es significativo y confiable.

$$
n=\frac{1,067}{1+\frac{(1,067-1)}{150}}=132 \text { muestras }
$$

Ecuación 2. Sustitución del tamaño de la muestra estadística. 
Después de obtener las mediciones para las características de calidad de la bobina eléctrica se procede al análisis de capacidad del proceso. Para el ancho, las mediciones se distribuyen de manera normal como lo indica el valor- $p=0.876$, en la gráfica de control para valores individuales se puede observar como la media del proceso es mayor que el límite superior. La capacidad real del proceso por lo tanto, utilizando el índice $C_{p k}=-0.04$ no es satisfactoria para cumplir con los limites dimensionales, (ver Fig. 5).

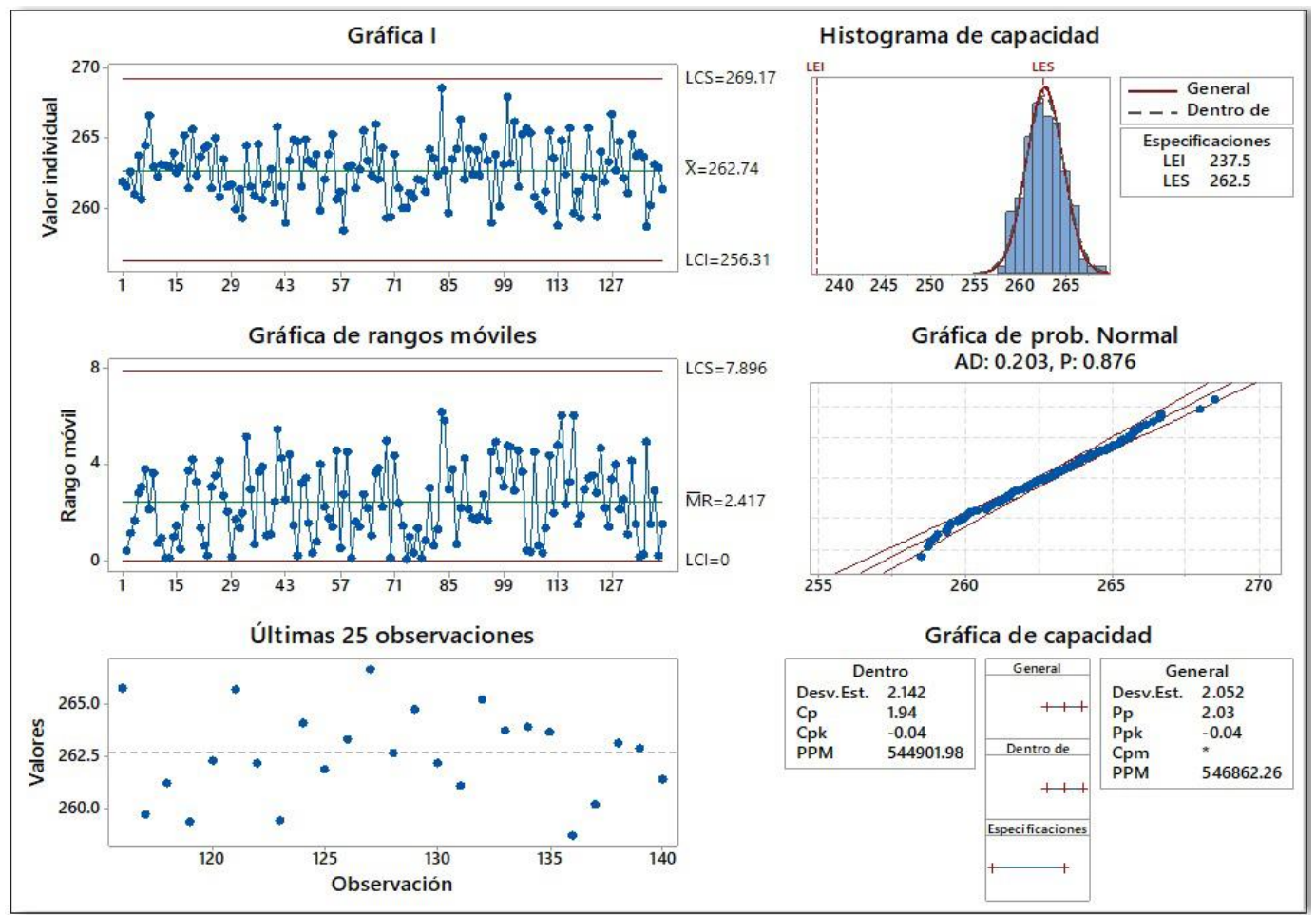

Fig. 5. Informe de capacidad para ancho.

Para el caso del largo, los datos también se distribuyen de manera normal y dentro de especificaciones, sin embargo, el índice $C_{p k}=1.19$, es susceptible de mejora sobre todo en el centrado del proceso, (ver Fig. 6). 


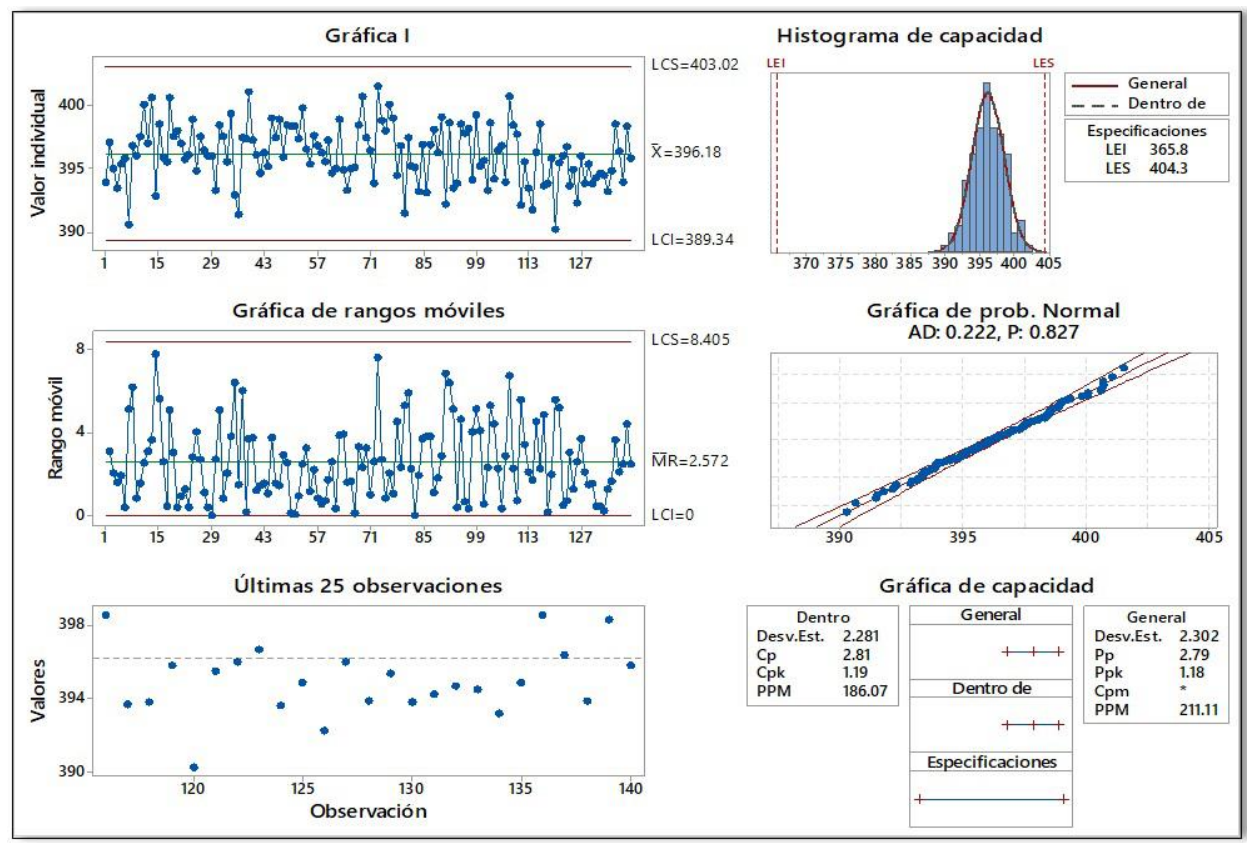

Fig. 6. Informe de capacidad para largo.

Finalmente, es posible concluir sobre las dimensiones de las bobinas de la siguiente manera; el lote de bobinas en cuanto a la variable de largo cumple con las especificaciones de diseño, sin embargo, se encuentra en la región cercana al límite de especificación superior. La variable de ancho presenta un $53 \%$ de bobinas que no cumplen con las especificaciones de diseño, representando un área de oportunidad. De acuerdo a lo anterior es importante definir medidas que lleven a mejorar la capacidad del proceso en la operación de ensamble.

\section{2) Diseño de experimentos}

La experimentación es un elemento clave en la ingeniería tanto en la etapa de diseño como en la mejora de cualquier proceso de fabricación, para ello es necesario realizar una serie de pruebas de las cuales se obtendrán datos que al ser analizados estadísticamente proporcionan la información para determinar los mejores niveles de los parámetros y así aumentar el desempeño de los procesos. En (Montgomery y Runger, 2014), se mencionan que el DOE consiste en realizar cambios deliberados en las variables controlables del proceso, se observan los resultados, se infiere y decide cuáles variables son responsables de los cambios en la salida.

Se llevó a cabo un diseño experimental para de determinar los parámetros apropiados de fabricación de las bobinas. El problema se presenta principalmente, en el ancho de la bobina, por ello, el equipo interdepartamental desarrolló un análisis a través de lluvia de ideas y la generación 
de diagramas causa-raíz con el método de las $6 M$; como resultado se detectaron causas potenciales prioritarias en las ramas de maquinaria y materiales, el factor considerado en maquinaria fue la tensión de alambre. La fuerza de tensión aplicada a las bobinas eléctricas es de suma importancia para lograr la calidad deseada en dos aspectos:

Dimensión de la bobina: Existe una relación directa entre el voltaje de la bobina primaria y secundaria de un transformador, este depende siempre del número de vueltas de alambre que tengan las dos bobinas del transformador. El número de vueltas realizadas en las bobinas es vital para el correcto desempeño del transformador por lo que alambre de cobre debe contener un grado de tensión tal que cumpla con el número de vueltas establecidas sin aumentar el tamaño de la bobina o que inclusive pueda imposibilitar el ensamble de la parte viva del trasformador, (Ruelas, 2018).

Disminución de fallas mecánicas en la bobina: Una de las fallas que pueden presentarse en las bobinas a consecuencia de colocar el cable de cobre con una inadecuada tensión es una falla mecánica en el transformador, generando una disminución de la eficiencia deseada. La falla ocurre por aflojamientos o desplazamientos ocasionados a falta de tensión en la fabricación de las bobinas, por otro lado, una excesiva tensión del cable en la manufactura de la bobina genera rupturas en el cable que en consecuencia también resulta en una falla mecánica, (Al-Janabi, 2015). En la Fig. 7 se muestra el factor de tensión del alambre de cobre durante la fabricación de la bobina eléctrica.

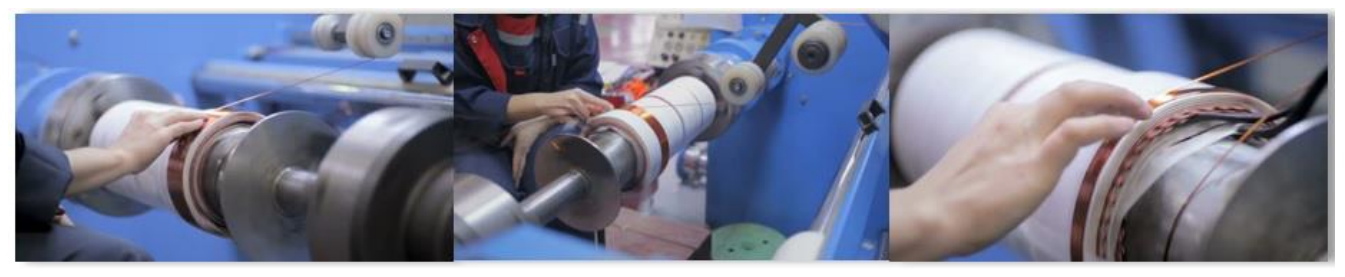

Fig. 7. Tensión del alambre.

Para la rama de material se consideró el factor de proveedor de papel dieléctrico. El papel dieléctrico es un aislante que debe garantizar una intensidad de campo eléctrico determinado al brindar tanto rigidez dieléctrica como mecánica a los conductores. Una condición importante para garantizar la rigidez dieléctrica y mecánica en el material conductor de la bobina es el espesor del papel, la bobina debe ser envuelta en capas de papel que garanticen la función de aislante lo 
que está ligado directamente con sus dimensiones (Jiménez, 2016). El papel utilizado en la experimentación se muestra en la imagen de la Fig. 8.
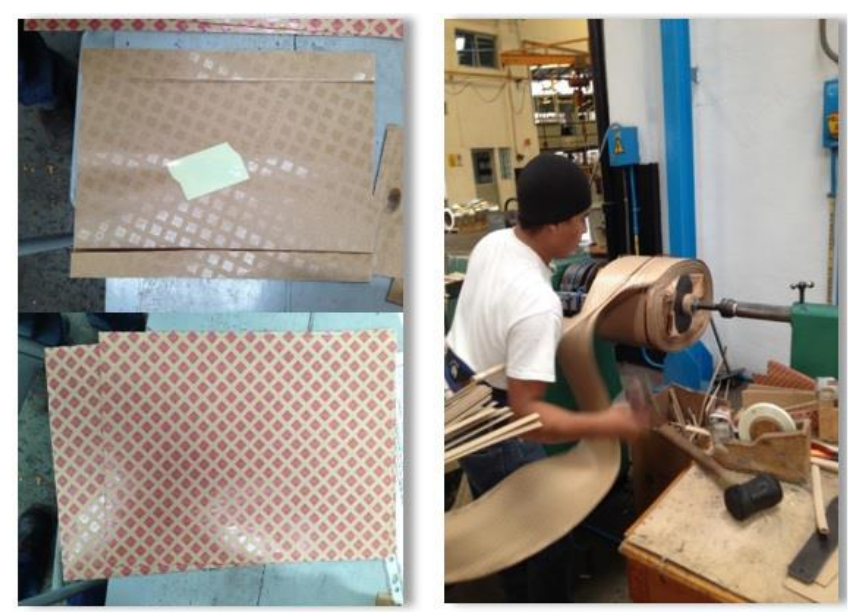

Fig. 8. Papel dieléctrico proveedor $\mathrm{W}$ y C.

El diseño experimental definido para su ejecución es un diseño Factorial $2^{2}$, en el cual serán analizados únicamente los factores tensión del cable y tipo de papel dieléctrico, mismo que afectan directamente las dimensiones de la bobina. Un principio de la metodología del diseño de experimental es la de llevar a cabo por lo menos una réplica del diseño, en el diseño experimental propuesto se llevan a cabo cuatro réplicas con el fin de obtener más datos de la variación en la variable de respuesta. La variable de respuesta queda establecida como la dimensión final en cuanto a largo y ancho de las bobinas eléctricas terminadas con característica menor es mejor, ya que a menores dimensiones, las operaciones de ensamble de parte viva y tanque podrán ser llevadas a cabo de forma eficiente. Sobre los niveles de los factores, el factor tipo de papel dieléctrico está limitado a la cantidad de proveedores avalados por la empresa fabricante de transformadores, en el caso del factor nivel de tensión mecánica sobre el alambre, las condiciones operacionales de la maquina empleada sólo opera en el modelo seleccionado a dos diferentes tensiones 20x10 y 40x10 Newtons, por lo que imposibilita agregar puntos centrales o emplear un diseño experimental mixto. Los factores considerados y sus niveles se presentan en la Tabla 3. 

experimental $2^{\mathrm{k}}$

Tabla 3. Factores y niveles experimentales.

\begin{tabular}{|ccc|}
\hline \multirow{2}{*}{ Factores } & \multicolumn{2}{c|}{ Niveles } \\
\cline { 2 - 3 } & Nivel Bajo (-) & Nivel Alto (+) \\
\hline Tensión (Newtons) & $2 \times 10$ & $4 \times 10$ \\
Proveedor de papel & $\mathrm{C}$ & $\mathrm{W}$ \\
\hline
\end{tabular}

\section{Resultados}

Se definió el orden de experimentación y el análisis estadístico a través del software Minitab, los resultados de las 16 corridas experimentales $\left(2^{2}\right.$ x $\left.4=16\right)$ se muestran en la Tabla 4.

Tabla 4. Matriz del diseño de experimentos.

\begin{tabular}{|cccccccc|}
\hline Orden Est & OrdenCorrida & PtCentral & Bloques & TENSIÓN & PAPEL & ANCHO & LARGO \\
\hline 3 & 1 & 1 & 1 & 2 & $\mathrm{~W}$ & 266 & 396 \\
14 & 2 & 1 & 1 & 4 & $\mathrm{C}$ & 264 & 397 \\
1 & 3 & 1 & 1 & 2 & $\mathrm{C}$ & 271 & 402 \\
11 & 4 & 1 & 1 & 2 & $\mathrm{~W}$ & 264 & 394 \\
9 & 5 & 1 & 1 & 2 & $\mathrm{C}$ & 273 & 403 \\
7 & 6 & 1 & 1 & 2 & $\mathrm{~W}$ & 265 & 398 \\
15 & 7 & 1 & 1 & 2 & $\mathrm{~W}$ & 270 & 401 \\
5 & 8 & 1 & 1 & 2 & $\mathrm{C}$ & 270 & 400 \\
13 & 9 & 1 & 1 & 2 & $\mathrm{C}$ & 269 & 401 \\
8 & 10 & 1 & 1 & 4 & $\mathrm{~W}$ & 262 & 392 \\
16 & 11 & 1 & 1 & 4 & $\mathrm{~W}$ & 259 & 389 \\
12 & 12 & 1 & 1 & 4 & $\mathrm{~W}$ & 261 & 390 \\
10 & 13 & 1 & 1 & 4 & $\mathrm{C}$ & 263 & 396 \\
6 & 14 & 1 & 1 & 4 & $\mathrm{C}$ & 265 & 397 \\
4 & 15 & 1 & 1 & 4 & $\mathrm{~W}$ & 258 & 388 \\
2 & 16 & 1 & 1 & 4 & $\mathrm{C}$ & 264 & 398 \\
\hline
\end{tabular}

Con el objetivo de analizar la variabilidad en el ancho de las bobinas del experimento se empleó el análisis de varianza (ANOVA). Se observa que los valores- $p$ de los dos factores son significativos, sin embargo, la interacción entre los factores no es significativa. El modelo obtenido de este diseño arrojó un coeficiente de determinación $R^{2}{ }_{a j}$ aceptable, por lo tanto, se podría asegurar que un $81.76 \%$ de la variación observada en el ancho de la bobina es explicada por el modelo. Además es posible aseverar que la tensión tiene un mayor efecto sobre el ancho de la bobina. Ver Tabla 5. 
Tabla 5. ANOVA Ancho.

\begin{tabular}{|lccccc|}
\hline \multicolumn{1}{|c}{ Fuente } & GL & SC & CM & Valor $\mathbf{F}_{\mathbf{0}}$ & Valor $\boldsymbol{p}$ \\
\hline Modelo & 3 & 241.500 & 80.500 & 23.28 & 0.000 \\
\hline Lineal & 2 & 241.250 & 120.625 & 34.88 & 0.000 \\
Tensión & 1 & 169.000 & 169.000 & 48.87 & 0.000 \\
Papel & 1 & 72.250 & 72.250 & 20.89 & 0.001 \\
\hline Interacciones dobles & 1 & 0.250 & 0.250 & 0.07 & 0.793 \\
$\quad$ Tensión*Papel & 1 & 0.250 & 0.250 & 0.07 & 0.793 \\
\hline Error & 12 & 41.500 & 3.458 & & \\
Total & 15 & 283.000 & & & \\
$\mathbf{R}^{\mathbf{2}=85.34 \%} \quad \mathbf{R}^{2}{ }_{\text {aj }}=81.76 \%$ & & & & \\
\hline
\end{tabular}

En la segunda variable de interés los cuadrados medios y el valor-p indican también que la interacción entre los factores no es significativa. El modelo obtenido de este diseño arrojó un coeficiente de determinación $R^{2}{ }_{a j}$ aceptable, la variabilidad presente en los datos del largo de la bobina es explicada por el modelo en un 83.79\%. Como en el ancho, el factor tensión aplicada al cable de cobre es más significativa que el factor papel. Ver Tabla 6.

Tabla 6. ANOVA Largo

\begin{tabular}{|lccccc|}
\hline \multicolumn{1}{|c}{ Fuente } & GL & SC & CM & Valor $\mathbf{F}_{\mathbf{o}}$ & Valor $\boldsymbol{p}$ \\
\hline Modelo & 3 & 285.250 & 95.083 & 26.85 & 0.000 \\
\hline Lineal & 2 & 276.250 & 138.125 & 39.00 & 0.000 \\
Tensión & 1 & 144.000 & 144.000 & 40.66 & 0.000 \\
Papel & 1 & 132.250 & 132.250 & 37.34 & 0.001 \\
\hline Interacciones dobles & 1 & 9.000 & 9.000 & 2.54 & 0.137 \\
Tensión*Papel & 1 & 9.000 & 9.000 & 2.54 & 0.137 \\
\hline Error & 12 & 42.500 & 3.542 & & \\
Total & 15 & 327.750 & & & \\
$\mathbf{R}^{2}=87.03 \%$ & $\mathbf{R}_{\text {aj }}^{\mathbf{2}}=83.79 \%$ & & & & \\
\hline
\end{tabular}

En la Fig. 9 se presentan las gráficas de cubos para las dos variables de interés (región experimental), donde se reporta lo predicho por el modelo en las combinaciones de los niveles de los dos factores. El mejor tratamiento para el largo de la bobina se predice en 389.75 milímetros y para el ancho en 260.00 milímetros. 
Estimación de parámetros operativos eficientes enfocados a la fabricación de bobinas eléctricas empleando un diseño experimental $2^{\mathrm{k}}$

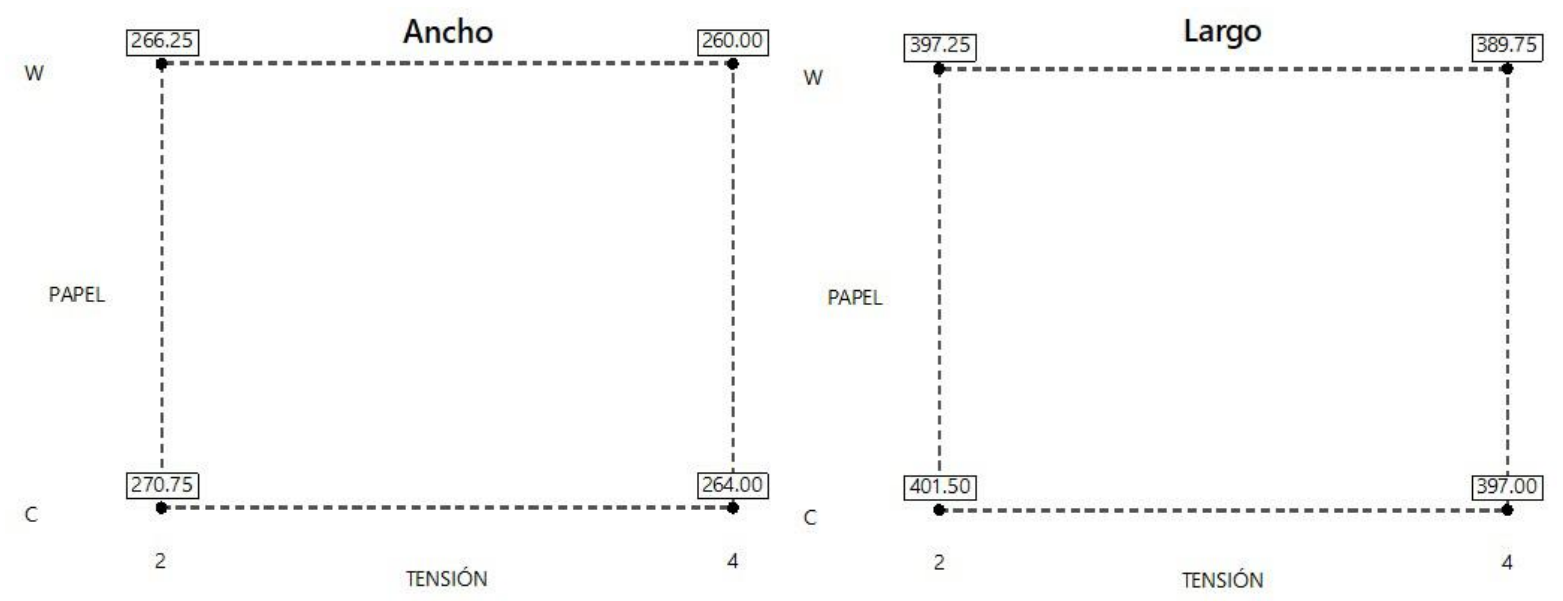

Fig. 9. Gráficas de cubos para ancho y largo.

Con el fin de lograr controlar las variables dimensionales de las bobinas eléctricas, se utilizó la gráfica de estimación presentada en la Fig. 10, en donde se obtiene una deseabilidad compuesta de 0.875 y se recomienda usar el nivel alto de la Tensión de alambre para embobinado $4 \times 10^{1} \mathrm{~N} \mathrm{y}$ el proveedor de papel dieléctrico W. Esta combinación de niveles permite durante la fabricación de bobinas las menores dimensiones.

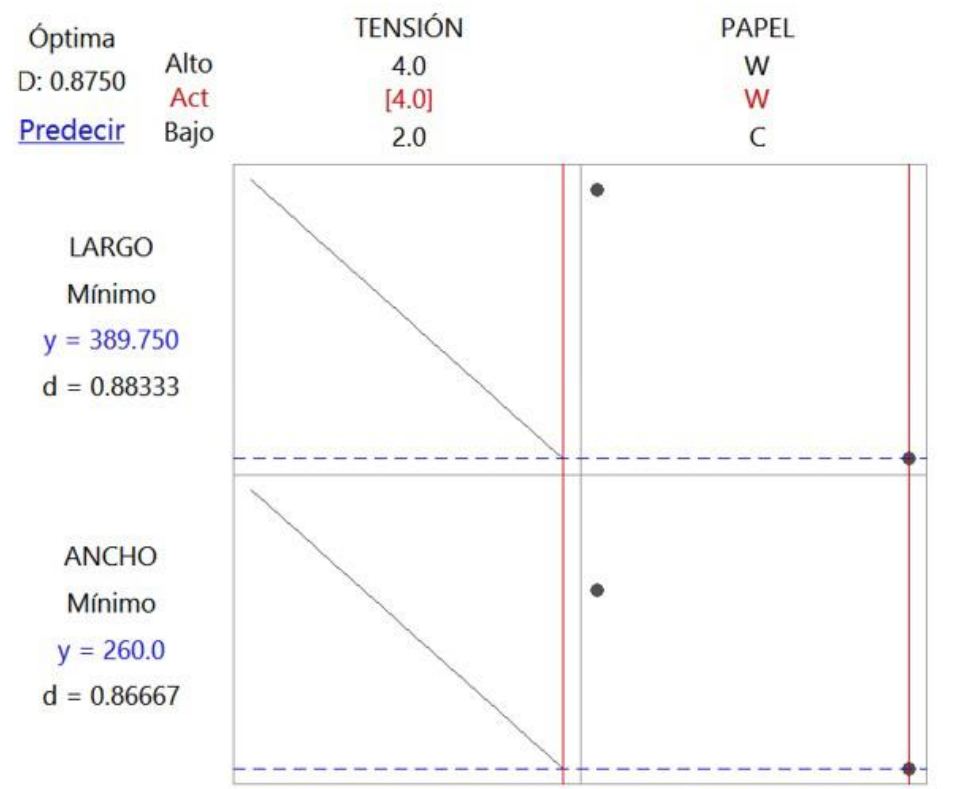

Fig. 10. Gráfica de estimación dentro de la región experimental.

Para complementar el análisis estadístico y comprobar la validez de los resultados se verificaron los supuestos del modelo, se puede observar en las gráficas de la Fig. 11 que para las dos 
variables los residuales se ajustan de manera adecuada a la recta, por lo cual, asumen una distribución normal, de la misma manera tanto la varianza constante como la independencia de los residuos se distribuyen sin ningún patrón contundente, por lo que no hay evidencias de falta de cumplimiento de cada uno de los supuestos estadísticos establecidos en la metodología del diseño experimental.
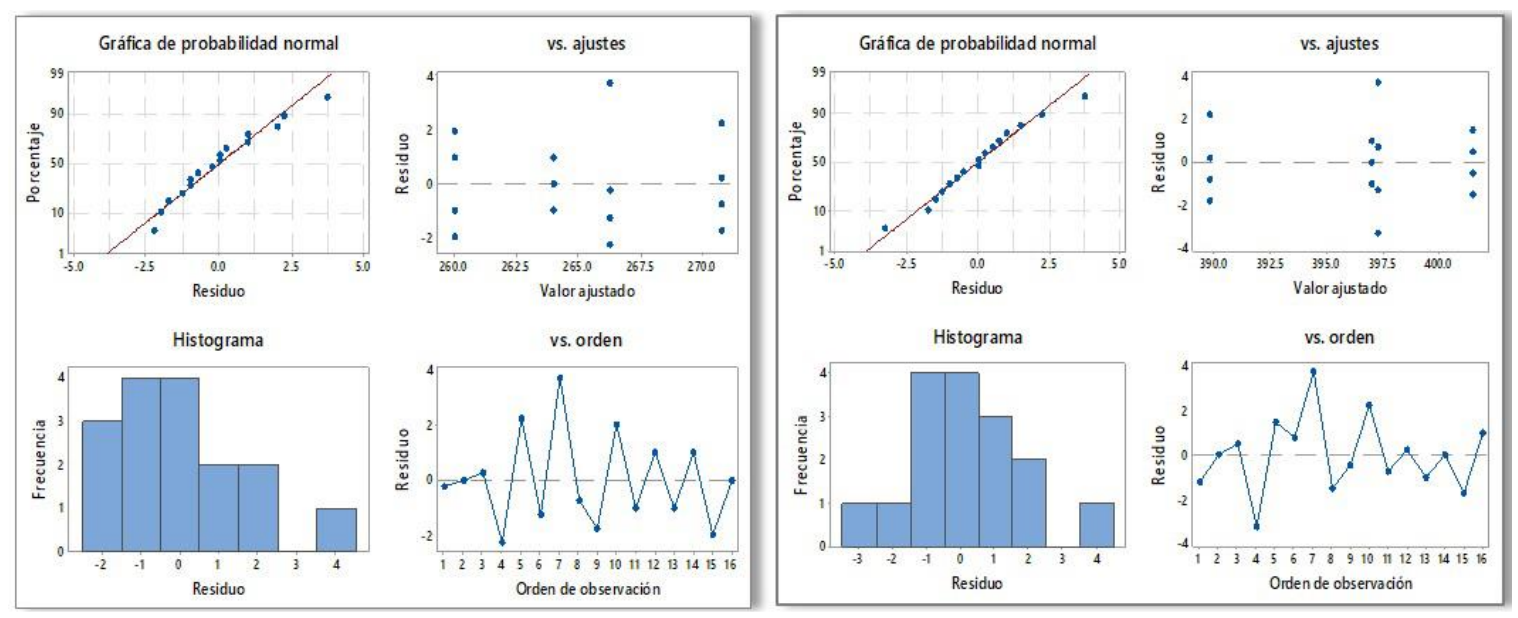

Fig. 11. Supuestos del modelo para largo y ancho.

\section{Análisis de capacidad final}

Con base en lo aprendido del proceso de fabricación de las bobinas fue posible realizar un lote de confirmación para llevar a cabo las mejoras necesarias al momento de ensamblar la parte activa, se fabricó un lote de 140 bobinas utilizando los niveles que permiten mejorar las dimensiones. Se evaluó la capacidad del proceso con el fin de determinar en qué medida el proceso cumple con las especificaciones dimensionales establecidas en la manufactura de las bobinas eléctricas.

El resultado del análisis de capacidad se presenta en la Fig. 12. Es posible observar la habilidad del proceso para cumplir con las especificaciones de $\pm 5 \%$ del ancho de la bobina, donde se presentaba principalmente la problemática, logrando una mejora significativa, se tenía un $53 \%$ del lote que no cumplían con tales especificaciones, con el presente estudio el $100 \%$ del lote de bobinas cumplen con los límites de especificación dimensionales, la variación por ende se reduce con una desviación estándar de 1.36. Por su parte el índice de capacidad real del proceso $C_{p k}=1.04$ indica la necesidad de trabajar en la mejora del centrado del proceso para alcanzar el mejor potencial, Otro punto que cabe resaltar es la presencia de dos puntos fuera de control 
Estimación de parámetros operativos eficientes enfocados a la fabricación de bobinas eléctricas empleando un diseño experimental $2^{\mathrm{k}}$

estadístico en la carta de control de rangos móviles, las señales de alerta sugieren seguir estudiando posibles ajustes que permitan reducir la variación y un centrado del proceso en la característica de calidad relacionada al ancho de bobina, con el fin de establecer un proceso más eficiente. Aunque cabe destacar que la presencia de señales fuera de control estadístico no determinan que existan piezas fuera de especificación.

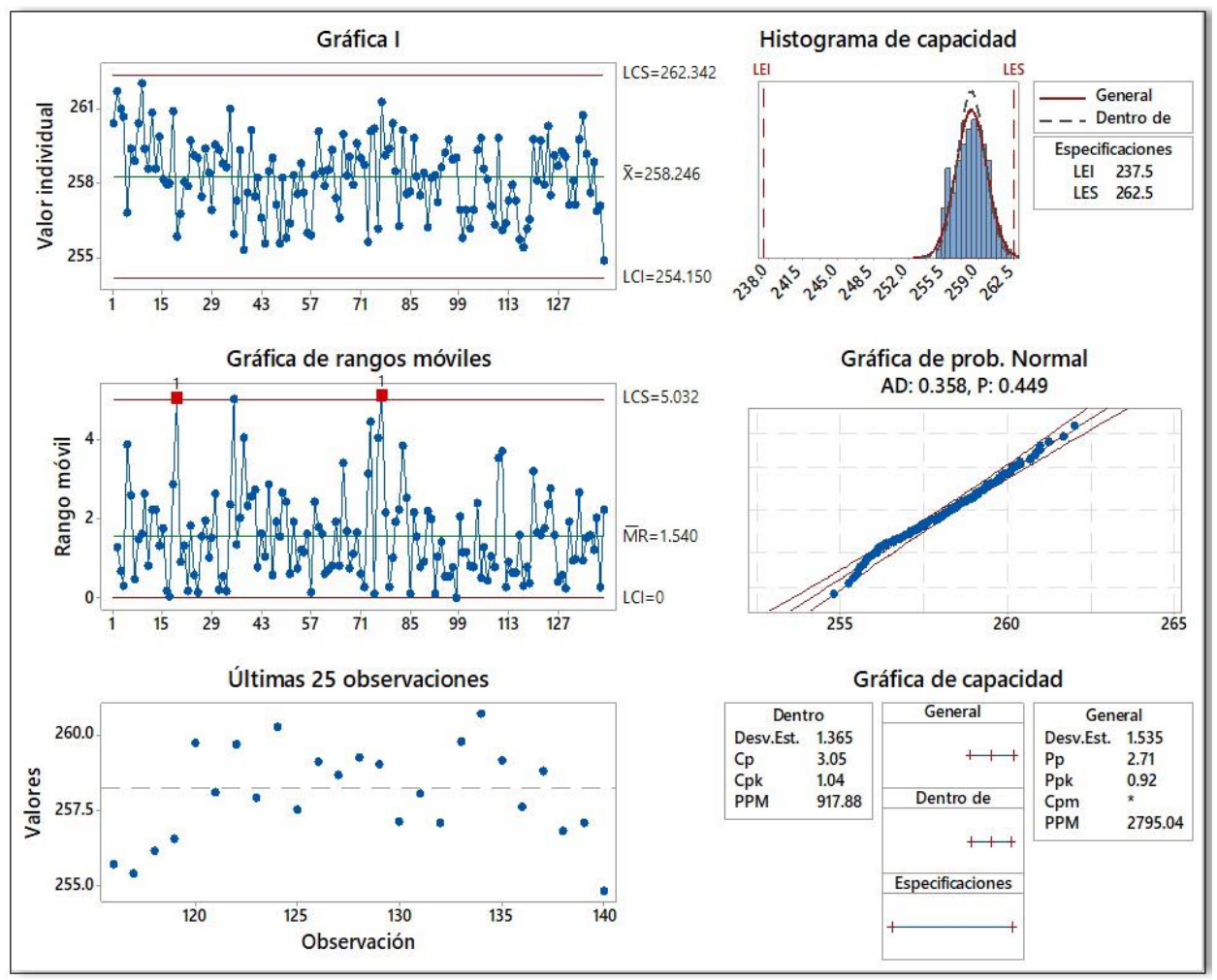

Fig. 12. Análisis de capacidad para ancho.

La variación y el centrado del proceso en la variable de largo de la bobina mejora de manera sustancial, anteriormente, las mediciones se encontraban muy cercanas al límite de especificación superior, así mismo, el índice de capacidad real del proceso con un valor de $C_{p k}=1.35$ indica un proceso con capacidad satisfactoria, como se aprecia en el histograma de la Fig. 13, el 100\% de las mediciones del largo se encuentran dentro de los límites de especificación. 


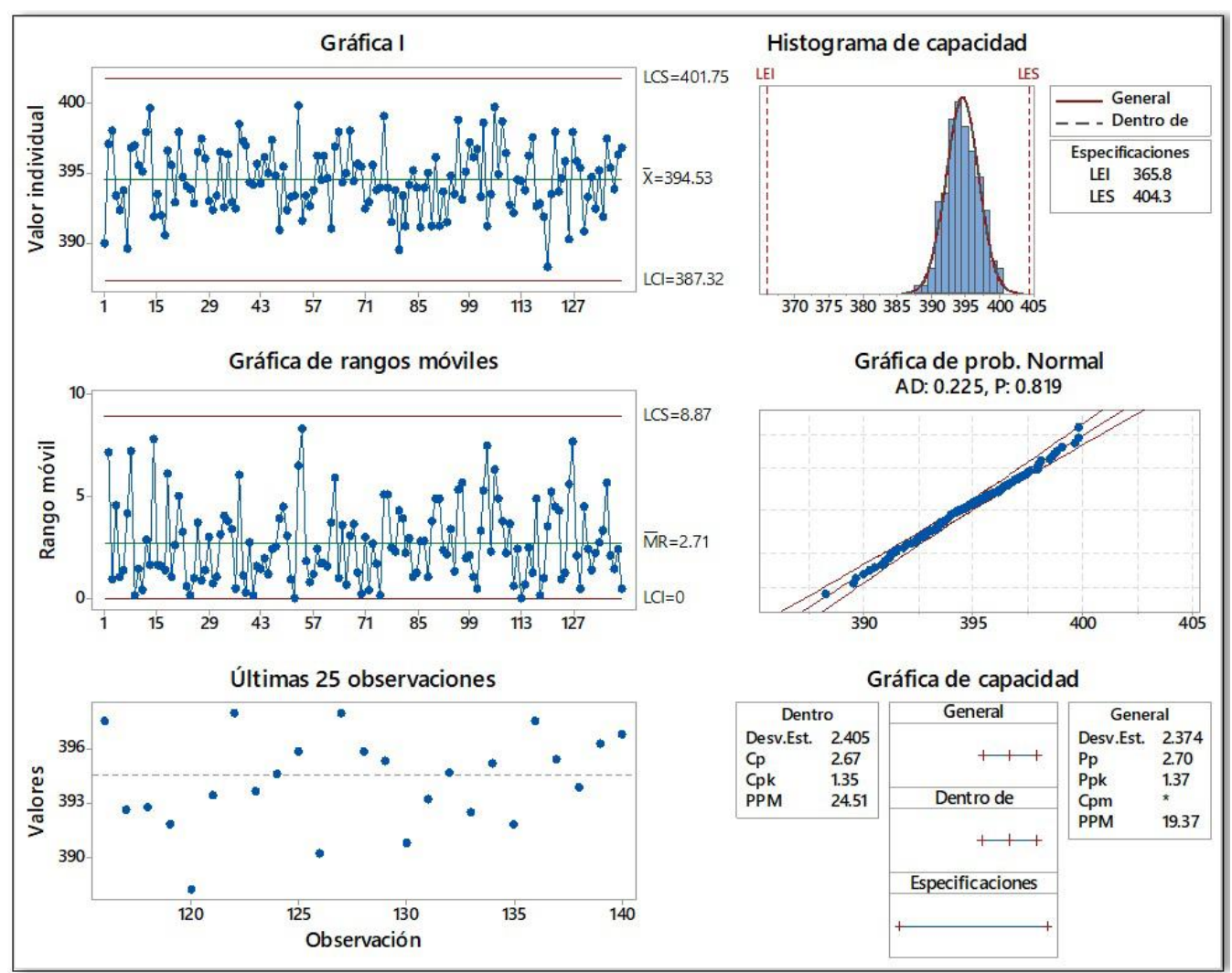

Fig. 13. Análisis de capacidad para largo.

El resumen de la evaluación del proceso antes y después de establecer las condiciones adecuadas de fabricación para las bobinas eléctricas para las dimensiones estudiadas se presenta en la Tabla 7, donde se reflejan los cambios y mejoras de manera significativa.

Tabla 7. Resumen capacidad del proceso.

\begin{tabular}{|cc|cc|}
\hline \multicolumn{2}{|c|}{ Ancho } & \multicolumn{2}{c|}{ Largo } \\
\hline Antes & Después & Antes & Después \\
$\mathbf{C}_{\mathbf{p k}}=\mathbf{- 0 . 0 4}$ & $\mathrm{C}_{\mathrm{pk}}=1.04$ & $\mathrm{C}_{\mathrm{pk}}=1.19$ & $\mathrm{C}_{\mathrm{pk}}=1.35$ \\
$\overline{\mathbf{X}}=\mathbf{2 6 2 . 7 4}$ & $\overline{\mathrm{X}}=258.24$ & $\overline{\mathrm{X}}=396.18$ & $\overline{\mathrm{X}}=394.53$ \\
$\mathbf{S}=\mathbf{2 . 1 4 2}$ & $\mathrm{S}=1.365$ & $\mathrm{~S}=2.281$ & $\mathrm{~S}=2.405$ \\
$\mathbf{P P M}=\mathbf{5 4 4 , 9 0 2}$ & $\mathrm{PPM}=918$ & $\mathrm{PPM}=186.07$ & $\mathrm{PPM}=24.55$ \\
\% Fuera de LE $=53 \%$ & \% Fuera de LE $=0 \%$ & \% Fuera de LE $=0 \%$ & $\%$ Fuera de LE $=0 \%$ \\
\hline
\end{tabular}




\section{Conclusión}

La importancia del sector eléctrico es primordial desde el punto de vista económico por su presencia en la mayoría de los procesos productivos, además de su necesidad para satisfacer los servicios básicos del consumidor final. Desde el enfoque de manufacturar productos empleados directamente en el sector eléctrico es vital poder establecer herramientas que permitan mejorar los procesos de fabricación, con el fin de mejorar la calidad del servicio y confiabilidad del sistema eléctrico.

La propuesta de este trabajo fue establecer condiciones de operación adecuadas para la manufactura eficiente de bobinas eléctricas que posibiliten un correcto ensamble de transformadores eléctricos. El estudio estadístico se centró en las variables con mayor influencia sobre las dimensiones de la bobina eléctrica, dichas variables de proceso son: tensión mecánica aplicada al alambre de cobre y tipo de papel dieléctrico, ambas variables influyen de forma estadísticamente significativa en las dimensiones de la parte viva del transformador y por consecuencia en su correcto ensamble.

El análisis de capacidad inicial permitió conocer el estado actual y aprender del proceso. Se utilizó un diseño experimental factorial donde se encontró que los niveles altos de papel y tensión permiten cumplir con los límites de especificación establecidos en el diseño del trasformador. Se logró mejorar el centrado del proceso en las dos variables de interés. La capacidad real del proceso se incrementó de manera significativa, principalmente, en la variable del ancho de la bobina.

Los métodos estadísticos utilizados en el presente trabajo fueron una herramienta eficaz para estudiar el proceso, la influencia de la tensión $(4 \times 10 \mathrm{~N})$ y del papel dieléctrico $(\mathrm{W})$ sobre la minimización de las dimensiones, mejorar la eficiencia del producto y los costos por re-trabajos. El estudio se realizó únicamente, para transformadores monofásicos tipo pedestal, sin embargo, es factible replicar el trabajo propuesto en otros procesos y tipos de transformadores que requieran establecer parámetros de fabricación adecuados en los sistemas productivos.

\section{$\underline{\text { Referencias }}$}

Al-Janabi, S., Rawat, S., Patel, A., \& Al-Shourbaji, I. (2015). Design and evaluation of a hybrid system for detection and prediction of faults in electrical transformers. International Journal of Electrical Power \& Energy Systems, 67, 324-335. 
Anteliz, A. (2012, September). Maintenance predictive in electric transformers, applying spectral analysis to the flow of magnetic dispersion. In Image, Signal Processing, and Artificial Vision (STSIVA), XVII Symposium, IEEE.

Della Giustina, D., Pau, M., Pegoraro, P. A., Ponci, F., \& Sulis, S. (2014). Electrical distribution system state estimation: measurement issues and challenges. IEEE Instrumentation \& Measurement Magazine, 17(6).

Dessouky, S., Ghoneim, S., Elfaraskoury, A., \& Sharaf, A. B. A. (2017). Barrier effect on the dielectric strength of the transformer insulating oils. In The 20th international symposium on high voltage engineering, Buenos Aires, Argentina, August, 1-5.

DOI: http://dx.doi.org/10.6036/8674

Dolan, A. I., \& Stefanescu, F. (2016, May). Exhaustive optimization method based on DOE and FEM applied on a SMES device. In Electrical Apparatus and Technologies (SIELA), 19th International Symposium IEEE, 1-4.

García, C. A., García, E., \& Villada, F. (2015). Implementación del Algoritmo Evolutivo MultiObjetivo de Frente de Pareto (SPEA) para la Planeación de Sistemas Eléctricos de Distribución incluyendo Huecos de Voltaje. Información tecnológica, 26(5), 155-168.

Gutiérrez Pulido, H., \& de la Vara Salazar, R. (2013). Control estadístico de la calidad y Seis Sigma. México DF: Mc Graw Hill Education.

Hydraulics \& Pneumatics, (2018), disponible en: http://www.hydraulicspneumatics.com/othercomponents/hydraulic-electric-analogies-part-6-coils-cores-and-transformers-0

Limón Portillo, Alejandro. (2017). La reorganización de la industria Eléctrica en México: Las consecuencias de la reforma energética. Centro de Investigación Económica y Presupuestaria, A.C.

M., Aravinthan, V., \& Pang, C. (2016, May). Life time analysis of distribution transformer using experimental design. In Transmission and Distribution Conference and Exposition (T\&D), 2016 IEEE/PES, 1-5.

Montgomery, D. C., \& Runger, G. C. (2014). Applied statistics and probability for engineers. John Wiley \& Sons.

Payan Tony, Z. S., \& C. D. J. R. (2016). Estado de derecho y reforma energética en México.

Petkovska, L., Digalovski, M., Cvetkovski, G., \& Lefley, P. (2014, September). A novel approach to multi-objective efficiency optimization for a distribution transformer based 
Estimación de parámetros operativos eficientes enfocados a la fabricación de bobinas eléctricas empleando un diseño experimental $2^{\mathrm{k}}$

on the Taguchi method. In Electrical Machines (ICEM), International Conference IEEE, pp. 2228-2234.

Phaengkieo, D., Somlak, W., \& Ruangsinchaiwanich, S. (2013, October). Transformer design by finite element method with DOE algorithm. In Electrical Machines and Systems (ICEMS), International Conference IEEE, 2219-2224.

Ranade, A., Rajkumar, E., \& Gaikwad, S. (2016). Transformer Tank Optimization using Design of Experiments. Indian Journal of Science and Technology, 9(34).

Ruelas-Santoyo, E., Vargas-Rodriguez, B., Cardiel-Ortega, J., \& Llamas-Perez, G. (2018). Function and development of multivariate control graphs. DYNA, 93(5). 493-496.

SENER (2017). Programa de desarrollo del sistema eléctrico nacional 2017-2031, disponible en: https://www.gob.mx/sener/acciones-y-programas/programa-de-desarrollo-del-sistemaelectrico-nacional-33462.

Zitouni, M., Guerbas, F., Boukezzi, L., \& Boubakeur, A. (2016). Modelling by design of experiments method of the AC breakdown voltage of transformer oil point-plane gaps with insulating barrier. IET Generation, Transmission \& Distribution, 10(1), 232-239. 